\title{
Spectral Efficiency Evaluation of 5G Waveform Candidates
}

\author{
Patteti Krishna, Tipparti Anil Kumar
}

\begin{abstract}
To increase the performance and to meet quality of service $(\mathrm{QoS})$ requirements of future wireless communication and networks, some of these approaches compete for incorporation in future wireless standards such as fifth generation (5G). In this paper comparative study and performance evaluation in terms of spectral efficiency (SE) their $P A P R$ of different $5 G$ waveform candidates. Most multicarrier schemes suffer from high PAPR and are not suitable when high energy efficiency is required.
\end{abstract}

Keywords: 5G, Spectral efficiency, PAPR, multicarrier schemes

\section{Introduction}

Fourth Generation (4G) wireless and mobile communication network has been rolled-out significantly and over a past few years high peak data rates has been increased dramatically[1]. Next generation wireless communication and networks should be improve key performance indicators such as peak data rates, energy and spectral efficiencies, massive connectivity, latency, mobility and power consumption significantly. New wireless network standard should be developed to reaching new services all above under the same networks [2]. The IMT2020[3] vision defines the fifth generation (5G) mobile communication to herald an era of truly immersive services. The 5G key performance service divided into three main categories such as enhanced mobile broadband (eMBB), massive machine-type communications (mMTC) and ultrareliable low-latency communications (URLLC) respectively [4]. To meet these requirements a new flexible 5G waveform candidate designed exactly [6].

This paper aims to provide a spectral efficiency analysis of $5 \mathrm{G}$ waveform discussions and overviews. The paper is organized as follows: Section II provides a brief discussion of the $5 \mathrm{G}$ waveform and waveform definition and requirements. Section III explains spectral efficiency and PAPR analysis of $5 \mathrm{G}$ and its comparisons along with the related advantages and disadvantages. Finally, Section IV concludes the paper.

\section{5G Fundamentals waveform}

Worldwide there are so many wireless groups working to define $5 \mathrm{G}$ needs/expectation, technology and other user requirements. An ideal $5 \mathrm{G}$ waveform should be fulfil the requirement of next generation wireless communications and networks such high spectral efficiency (SE), huge

Revised Manuscript Received on August 19, 2019.

Dr.Patteti Krishna,Department of Electronics and Communication Engineering, Jayamukhi Institute of Technological Sciences, Narsampet, Warangal, Telangana, India (kpatteti@gmail.com)

Dr.Tipparti Anil Kumar, Department of Electronics \& Communication Engineering, CMR Institute of Technology, Kandlakoya (v), Medchal Road, Hyderabad - 501401. (tvakumar2000@yahoo.co.in) amount of peak data rates, low latency, power consumption andout-of-band emission (OOB).Furthermore, to achieve robust against to wireless frequency fading channel.

5G wireless communication \& networks addresses various challenges in truly networked society such as the massive connectivity, high traffic and significantly increasing wide range of wireless applications with varying of wireless channel characteristics.

\section{Spectral Efficiency and PAPR Comparison of $5 G$ waveforms\& RESULTS}

In this section, we evaluated the performance comparison of $5 \mathrm{G}$ waveform candidates in terms of SE and their PAPR.

\section{A. Spectral Efficiency(SE)}

Firstly, compare the $5 \mathrm{G}$ waveforms in terms of SE versus the time duration of the burst. In this paper, we consider theLong-Term Evolution (LTE) system channel bandwidth $10 \mathrm{MHz}$ and its related used parameters shown in Table1. In simulations results, considered two users for asynchronous mode multiuser access technique.

The multi carrier modulation schemes [7] such as OFDM, FBMC, SC-FDMA, GFDM and UFMC, the SE does not depend on the burst time and is a function of FFT size, order of the modulation and the modulation parameters.

The OFDM and SC-FDMA have same spectral efficiency defined as

$$
\eta_{O F D M}=\eta_{S C-F D M A}=m \times \frac{N_{F F T}}{\left(N_{F F T}+N_{c p}\right)}
$$

where $m$ is the modulation order. For UFMC, SE losses because of transient state of the shaping filter.

The SE of UFMC is expressed as

$$
\eta_{U F M C}=m \times \frac{N_{F F T}}{\left(N_{F F T}+L-1\right)}
$$

The rest of the paper, we choose $\mathrm{L}=\mathrm{N}_{\mathrm{cp}}+1$, in order to have the same SE between UFMC and OFDM.

For GFDM, the CP insertion is done per symbol and the $\mathrm{SE}$ is expressed as

$$
\eta_{G F D M}=\frac{m \times K \times M}{m \times K+N_{c p}}
$$

The spectral efficiency (SE) of UFMC and OFDM has same, whereas the SE of GFDM depends on FFT size. The SE of FBMC [6]determined by on burst duration, if burst 
duration is always greater than $3 \mathrm{~ms}$, better SE than UFMC and OFDM [8].

Table 1: Simulation parameters

\begin{tabular}{|l|l|l|}
\hline \multicolumn{3}{|c|}{ Overall parameters } \\
\hline FFT size & $N_{F F T}$ & 1024 \\
\hline Bit per Symbol & $M$ & 2 \\
\hline Resource block size & $N_{R B}$ & 12 \\
\hline No. of RBs & $N_{\mathrm{Re}}^{1}$ & 3 for UE 1 \\
& $N_{\mathrm{Re}}^{2}$ & for UE2 \\
\hline Sampling frequency & $F_{e}$ & $15.36 \mathrm{MHz}$ \\
\hline
\end{tabular}

\begin{tabular}{|l|l|c|}
\hline \multicolumn{3}{|c|}{ OFDM and SC-FDMA } \\
\hline $\mathrm{CP}$ & $N_{C P}$ & 72 samples \\
\hline \multicolumn{2}{|c|}{ UFMC } \\
\hline Length of filter & $\mathrm{L}$ & 73 \\
\hline $\begin{array}{l}\text { Attenuation of Stop } \\
\text { band }\end{array}$ & $40 \mathrm{~dB}$ \\
\hline
\end{tabular}

\begin{tabular}{|l|l|c|}
\hline \multicolumn{2}{|c|}{ GFDM } \\
\hline Sub symbols & $P$ & 15 \\
\hline FFT size & M & 1024 \\
\hline Roll Off factor & $\alpha$ & 0.1 \\
\hline \multicolumn{2}{|c|}{ FBMC } \\
\hline Spreading factor & K & 4 \\
\hline
\end{tabular}

\begin{tabular}{|l|l|}
\hline \multicolumn{2}{|c|}{ Asynchronous access } \\
\hline Guard carriers & {$[1,2,4,5]$} \\
\hline Timing Offset & {$[-0.25: 0.25]$} \\
\hline CFO & $0 ; 10 \%$ \\
\hline
\end{tabular}

If we compare the SE's of GFDM and OFDM:

1. GFDM SE is higher than OFDM SE if the frequency grid is fixed (i,e., same number of data carrier and same FFT size). Each GFDM symbol contains then more modulated complex samples $\left(\mathrm{M} \times \mathrm{N}_{\mathrm{s}}\right.$ for $\mathrm{GFDM}$ versus $\mathrm{N}_{\mathrm{s}}$ for OFDM) and the size of a GFDM sub symbol is equal to the size of an OFDM symbol (without the CP insertion). SE increase for GFDM is due to the use of one CP per $\mathrm{M}$ sub symbols.

2. The GFDM SE is identical to the OFDM SE if we consider a constant data block size (i,e., $N_{S} \times M=N_{S}^{O F D M}$ and $K M=N_{F F T}$. It means that the frequency grid is modified, and divided by $M$ ). In such a case, the GFDM symbol size is the same as the OFDM one (the sub symbol size is thus $1 / \mathrm{M}$ compared to OFDM symbol size), and the frequency spacing is $\mathrm{M}$ times higher.

In this case, we have fixed the frequency grid, which means that the GFDM SE is thus higher than the OFDM SE.

For FBMC the SE depends on the frame duration. The SE loss is due to the transient state of the global shaping filter. Thus there is no constant loss per symbol (compared to other waveforms) and the SE increases with the burst duration to reach an asymptotic level equal to the modulation order.

The SE of FBMC is expressed as

$$
\eta_{F B M C}=\frac{m \times s \times N_{F F T}}{\frac{(2 s-1) N_{F F T}}{2}+N_{F F T} K}=\frac{m s}{s+K-\frac{1}{2}}
$$

where $\mathrm{S}$ denotes the number of symbols.

We compute the SE of the different waveform candidates, versus the duration of the burst. Results are depicted in Figure 1 with $m=2$. It is shown that the same SEs of UFMC andOFDM, the GFDM has a better SE compared to OFDM and UFMC. The SE loss for GFDM is low as the CP is added only once per symbol which means that there is $\mathrm{M}$ times less than CP for GFDM compared to OFDM. Besides, FBMC SE depends on the time duration, and is better than OFDM and UFMC if the burst duration is longer than 3.5 $\mathrm{ms}$ (when $\mathrm{K}=4$ and $m=2$ ).It asymptotically reaches the modulation spectral efficiency and is comparable to GFMD if the burst duration is higher than $18 \mathrm{~ms}$.

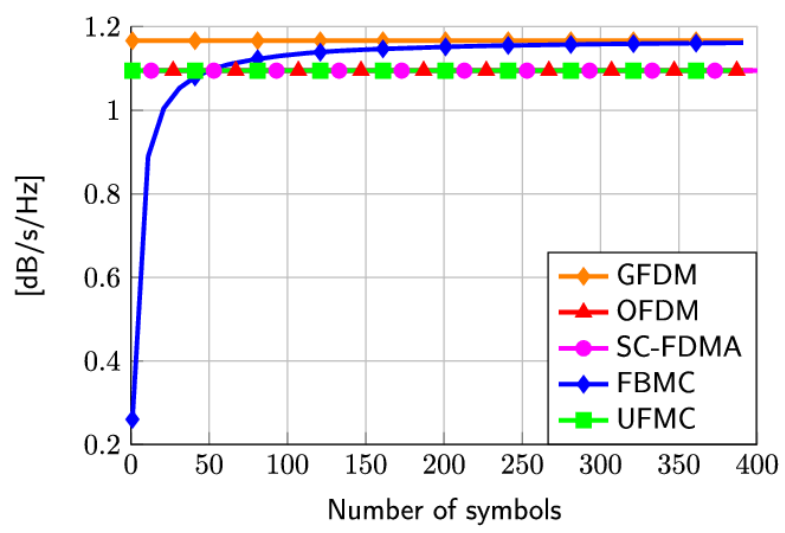

Fig.1: Spectral Efficiency versus number of symbols

\section{B. PAPR Comparison}

We compute the CCDF of the PAPR for the considered waveforms, for burst duration of $3 \mathrm{~ms}$ and with the parameters described in Table-1 with QPSK modulation. The PAPR is defined as

$$
P A P R=\frac{\max \left(|y[k]|^{2}\right)}{E\left[|y[k]|^{2}\right]}
$$

Figure 2 illustrated that the CCDF of PAPRversus PAPR of multicarrier modulation techniques. The SC-FDMA offers the best performance because of single carrier property and the other modulations have high PAPR. 


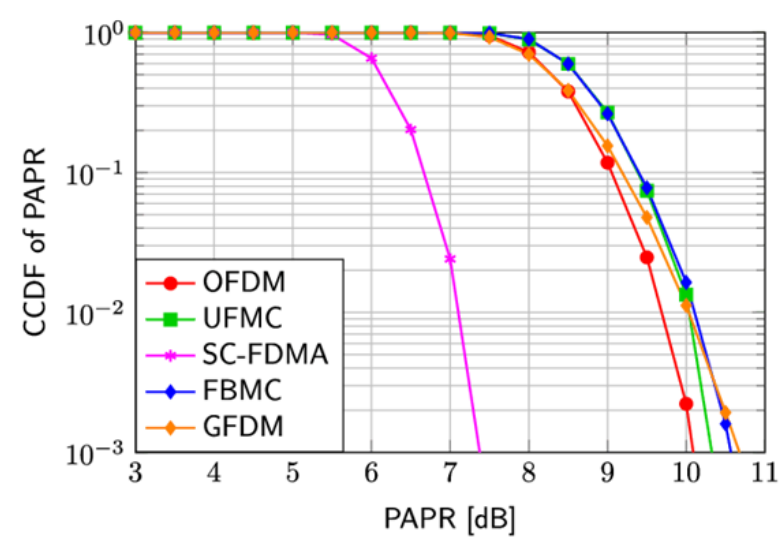

Fig.2: CCDF of PAPR

A summary of the main advantages/disadvantages of these major $5 \mathrm{G}$ candidate waveforms is provided in Table II.

Table II: The 5G waveform candidates.

\begin{tabular}{|c|c|c|}
\hline \multicolumn{3}{|c|}{ Multicarrier Schemes } \\
\hline Waveform & Advantages & Disadvantages \\
\hline OFDM & \begin{tabular}{|ll}
$\checkmark$ & Simple FDE \\
$\checkmark$ & Easy MIMO \\
& integration \\
$\checkmark$ & Flexible frequency \\
& assignment \\
$\checkmark$ & Low \\
& implementation \\
& complexity
\end{tabular} & \begin{tabular}{|ll}
$\checkmark$ & High OOBE and \\
& PAPR \\
$\checkmark$ & Strict \\
& synchronization \\
& requirement \\
$\checkmark$ & Poor performance \\
& for high mobility \\
& applications
\end{tabular} \\
\hline GFDM & $\begin{array}{ll}\checkmark & \text { Flexible design } \\
\checkmark & \text { Good frequency } \\
& \text { localization } \\
\checkmark & \text { Reduced PAPR }\end{array}$ & 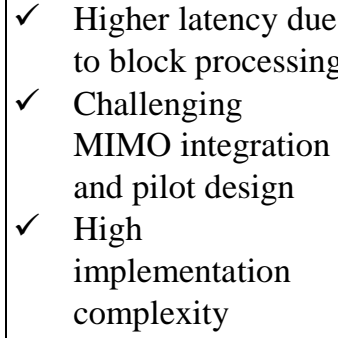 \\
\hline UFMC & \begin{tabular}{|ll}
$\checkmark$ & Good frequency \\
& localization \\
$\checkmark$ & Shorter filter \\
& length compared \\
& to subcarrier-wise \\
& operations (i.e., \\
& OQAM-FBMC \\
& and GFDM ) \\
$\checkmark$ & Compatible with \\
& MIMO
\end{tabular} & \begin{tabular}{|ll}
$\checkmark$ & No immunity to \\
& ISI due to lack of \\
& CP \\
$\checkmark$ & High receiver \\
& complexity due to \\
& increased FFT size
\end{tabular} \\
\hline FBMC & 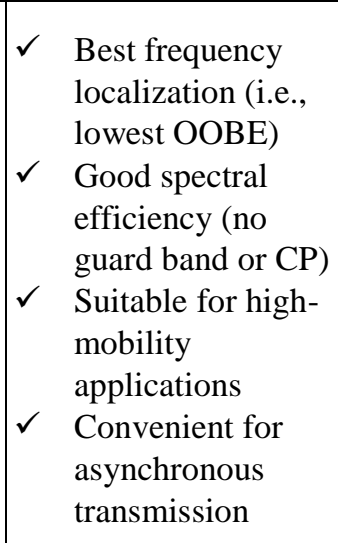 & \begin{tabular}{|ll}
$\checkmark$ & Challenging \\
& MIMO integration \\
& and pilot design \\
$\checkmark$ & No immunity to \\
& ISI due to lack of \\
& CP \\
$\checkmark$ & High \\
& implementation \\
& complexity \\
$\checkmark$ & Increased power \\
& consumption due \\
& to OQAM \\
& signaling
\end{tabular} \\
\hline
\end{tabular}

\section{CONCLUSIONS}

In this paper, discussed all waveforms for $5 \mathrm{G}$ provide lower OOBE compared to OFDM and SC-FDMA. The subcarrier-wise filtering operation in FBMC results in the best frequency localization among the candidate waveforms due to the use of longer filter lengths. Although GFDM is another subcarrier-wise filtered waveform, the rectangular window shape in the time domain causes abrupt transitions and increases OOBE. Most multicarrier schemes suffer from high PAPR and are not suitable when high energy efficiency is required. However, GFDM exhibits a reduced PAPR characteristic due to its equivalency to DFT-spread waveforms. The single-carrier schemes are preferable in energy-limited use cases along with the use of flexible guard intervals that provide better spectral confinement and improved PAPR. The spectral efficiency is another critical design criterion that is highly affected by the window/filter duration, the shape of filter, and extra overheads.

\section{REFERENCES}

1. Patteti Krishna, "Spectral Efficiency Analysis of Multicarrier Scheme for 5G Communications", International Journal of Recent Technology and Engineering (IJRTE) Volume-7, Issue-6, March 2019, pp: 1682-1686.

2. Qualcomm Inc., "Waveform Requirements," 3GPP Standard Contribution (R1-162198), Busan, Korea, Apr. 11-15 2016.

3. ITU-R, "IMT Vision - Framework and overall objectives of the future development of IMT for 2020 and beyond," Technical Report M.2083-0, Sept. 2015.

4. X. Zhang, L. Chen, J. Qiu, and J. Abdoli, "On the Waveform for 5G,” IEEE Commun. Mag., vol. 54, no. 11, pp. 74-80, 2016.

5. Patteti Krishna, "BER Performance evaluation of FBMCOAQM and OFDM Multicarrier system', International Journal of Innovative Technology and Exploring Engineering (IJITEE), Volume-8 Issue-10, August 2019, pp: 1564-1568.

6. Huawei, HiSilicon, "5G waveform: requirements and design principles," 3GPP Standard Contribution (R1-162151), Busan, Korea, Apr. 11-15 2016.

7. A.Sahin, I. Guvenc, and H. Arslan, "A Survey on Multicarrier Communications: Prototype Filters, Lattice Structures, and Implementation Aspects", IEEE Communications Surveys Tutorials, vol. 16, no. 3, pp. 1312-1338, March 2014.

8. B. Farhang-Boroujeny and H. Moradi, "OFDM Inspired Waveforms for 5G", IEEE Communications Surveys Tutorials, vol. 18, no. 4, pp. 2474-2492, Fourthquarter 2016 